7th International Workshop on Astronomy and

Relativistic Astrophysics (IWARA 2016)

International Journal of Modern Physics: Conference Series

Vol. 45 (2017) 1760054 (4 pages)

(C) The Author(s)

DOI: $10.1142 / \mathrm{S} 2010194517600540$

\title{
A Heavy-Light Quark Potential
}

\author{
Frederico F. Gomes, Bruna C. Folador, Dimiter Hadjimichef \\ Instituto de Física \\ Universidade Federal do Rio Grande do Sul (UFRGS) \\ Av. Bento Gonçalves, 9500, 91501-970 Porto Alegre, RS, Brazil \\ frederico.fetter@gmail.com, bru.folador@gmail.com, dimiter.hadjimichef@ufrgs.br \\ Daniel T. da Silva \\ Instituto de Física e Matemática \\ Universidade Federal de Pelotas (UFPel) \\ Rua dos Ipês, Capão do Leão, 96050-500 Pelotas, RS, Brazil \\ neodts@gmail.com
}

Published 15 August 2017

\begin{abstract}
In many studies of meson-baryon interactions with short one gluon exchange potential (OGEP), usually a full non-relativistic reduction, at the quark level Hamiltonian, is performed. In systems like $\eta_{c} N$, light and heavy quarks are present, which in principle would require only a partial non-relativistic reduction. We shal start from a JKJ relativistic quark Hamiltonian and apply a partial non-relativistic reduction in order to obtain a OGEP between heavy and light quarks (heavy-light quark potential).
\end{abstract}

Keywords: Quark Model, Relativistic Approximation, Meson-Nucleon Scattering.

PACS numbers: 12.39.Pn, 12.40.-y, 13.90.+i

\section{1. $\eta_{c} N$ Interaction}

Due to difficulties in dealing with non-perturbative aspects of QCD such as color confinement and the necessity to attent to the internal structure of hadrons to properly deal with several systems, effective quark models were developed. One such approach is the Fock-Tani formalism, in which a change of representations maps the physical Hilbert space into an ideal Hilbert space, where the bound-states are represented by "ideal particles" whose elementary creation and annihilation operators obey canonical commutation relations. The information about the hadrons internal structure is transfered to an effective Hamiltonian ${ }^{1-3}$. This formalism was shown to be equivalent, in lower orders, to the Resonating Group Method (RGM).

This is an Open Access article published by World Scientific Publishing Company. It is distributed under the terms of the Creative Commons Attribution 4.0 (CC-BY) License. Further distribution of this work is permitted, provided the original work is properly cited. 

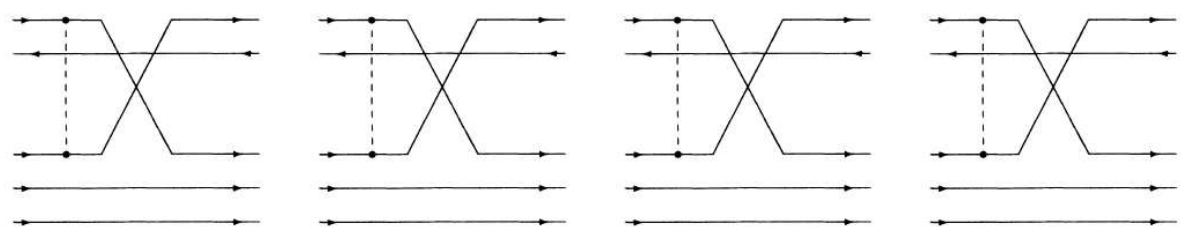

Fig. 1. One Gluon Exchange Potential scattering diagrams.

In particular the $\eta_{c} N$ system is formed by a mesons $\eta_{c}(c \bar{c})$ and the nucleon $N$ $(q q q)$, which in terms of the RGM scattering diagrams, with a One Gluon Exchange Potential (OGEP), can be seen in Fig. 1.

In the $\eta_{c} N$ interaction, quark interchange is associated with a one gluon exchange between constituent quarks. Traditionally, OGEP is obtained from a relativistic two-body quark interaction, taking an expansion in powers of the momentum of the constituent spinors, obtaining a non-relativistic approximation in the lowest order of momentum ${ }^{4}$. In the $\eta_{c} N$ system the $\eta_{c}$-meson has a pair of heavy quarks which will interact with the quarks from the nucleon. In this case the corresponding expansion should consider the interaction of a light relativistic quark with a heavy non-relativistic quark. The Cornell $J K J^{5}$ type relativistic quark interaction Hamiltonian is

$$
H_{I}=\frac{1}{2} \sum_{a=1}^{8} \mathcal{F}^{a} \mathcal{F}^{a} \int \mathrm{d} \vec{x} \mathrm{~d} \vec{y} J^{\mu}(\vec{x}) K_{\mu \nu}(\vec{x}-\vec{y}) J^{\nu}(\vec{y}),
$$

where

$$
J^{\mu}(\vec{x})=\bar{q}(\vec{x}) \gamma^{\mu} q(\vec{x}),
$$

are the quark currents, $\mathcal{F}^{a}=\lambda^{a} / 2$ are the generators of $S U(3)$ and the gluon potential $K_{\mu \nu}$, in the momentum space, is

$$
K_{00}(\vec{q})=-\frac{D_{0}}{q^{2}} \quad, \quad K_{i j}(\vec{q})=\frac{D_{0}}{q^{2}}\left(\delta_{i j}-\frac{q_{i} q_{j}}{q^{2}}\right) ;
$$

$K_{0 j}(\vec{q})=K_{i 0}(\vec{q})=0$ and $D_{0}=4 \pi \alpha_{s}$. After using Eq. (2), the basic structure of Eq. (1) reduces to

$$
J^{\mu} K_{\mu \nu}(\vec{q}) J^{\nu}=-\frac{D_{0}}{q^{2}} J^{0} J^{0}+\frac{D_{0}}{q^{2}} \vec{J} \cdot \vec{J}-\frac{D_{0}}{q^{2}}(\vec{J} \cdot \vec{q})(\vec{J} \cdot \vec{q}) .
$$

The spinor can be written as

$$
u_{s}(\vec{p})=\left(\begin{array}{c}
f(\vec{p}) \chi_{s} \\
g(\vec{p}) \vec{\sigma} \cdot \vec{p} \chi_{s}
\end{array}\right) \quad, \quad v_{s}(\vec{p})=\left(\begin{array}{c}
g(\vec{p}) \vec{\sigma} \cdot \vec{p} \chi_{s}^{c} \\
f(\vec{p}) \chi_{s}^{c}
\end{array}\right)
$$


where

$$
f(\vec{p})=\sqrt{\frac{E_{p}+m}{2 E_{p}}} \quad, \quad g(\vec{p})=\frac{1}{E_{p}+m} \sqrt{\frac{E_{p}+m}{2 E_{p}}},
$$

and $\chi_{i}, \chi_{i}^{c}$ are the Pauli spinors and where $E_{p}=\sqrt{p^{2}+m^{2}}$.

\section{Heavy-Light Potential}

The calculation will be restricted to the spin-spin interaction, which implies that the only relevant term is the second term in Eq. (3). Considering momentum conservation and defining $J_{s_{1} s_{2}}^{i}(\vec{p}+\vec{q}, \vec{p})$ the "heavy current" and $J_{s_{3} s_{4}}^{j}\left(\vec{p}^{\prime}-\vec{q}, \vec{p}^{\prime}\right)$ as the "light current", with $f_{i}=f\left(\vec{p}_{i}\right)$ and $g_{j}=g\left(\vec{p}_{j}\right)$, one has the relevant part of $J^{i}$ is

$$
\begin{aligned}
J_{s_{1} s_{2}}^{i}(\vec{p}+\vec{q}, \vec{p}) & =i\left(f_{1} g_{2}-f_{2} g_{1}\right)(\vec{\sigma} \times \vec{p})^{i}+f_{2} g_{1}\left[\delta_{12} q^{i}+i(\vec{\sigma} \times \vec{q})^{i}\right] \\
J_{s_{3} s_{4}}^{j}\left(\vec{p}^{\prime}-\vec{q}, \vec{p}^{\prime}\right) & =i\left(f_{3} g_{4}-f_{4} g_{3}\right)\left(\vec{\sigma}^{\prime} \times \vec{p}^{\prime}\right)^{j}-f_{4} g_{3}\left[\delta_{34} q^{j}+i\left(\vec{\sigma}^{\prime} \times \vec{q}^{\prime}\right)^{j}\right] .
\end{aligned}
$$

The heavy current contribution of order $J_{s_{1} s_{2}}^{i} \sim p$, which implies that $f_{1} g_{2}-$ $f_{2} g_{1} \approx 0$ and $f_{2} g_{1} \approx 1 / 2 m_{p}$, resulting in

$$
J_{s_{1} s_{2}}^{i}(\vec{p}+\vec{q}, \vec{p})=\frac{i}{2 m_{p}}(\vec{\sigma} \times \vec{q})^{i} .
$$

The light current is $J_{s_{3} s_{4}}^{j} \sim p^{3}$ and is obtained by similar procedure:

$$
\begin{aligned}
J_{s_{3} s_{4}}^{j}\left(\vec{p}^{\prime}-\vec{q}, \vec{p}^{\prime}\right)=i & {\left[\frac{q^{2}}{8 m_{l}^{3}}-\frac{\vec{p}^{\prime} \cdot \vec{q}}{4 m_{l}^{3}}\right]\left(\vec{\sigma}^{\prime} \times \vec{p}^{\prime}\right)^{j} } \\
& -i\left[\frac{1}{2 m_{l}}-\frac{p^{2}}{4 m_{l}^{3}}+\frac{3 \vec{p}^{\prime} \cdot \vec{q}}{8 m_{l}^{3}}-\frac{3 q^{2}}{16 m_{l}^{3}}\right]\left(\vec{\sigma}^{\prime} \times \vec{q}\right)^{j} .
\end{aligned}
$$

The spin-spin contribution [second term in Eq. (3)] for the $Q q$ system is

$$
\begin{aligned}
\vec{J}_{s_{1} s_{2}} \cdot \vec{J}_{s_{3} s_{4}}= & -C_{1}\left[\left(\vec{\sigma} \cdot \vec{\sigma}^{\prime}\right)\left(\vec{q} \cdot \vec{p}^{\prime}\right)-\left(\vec{\sigma} \cdot \vec{p}^{\prime}\right)\left(\vec{\sigma}^{\prime} \cdot \vec{q}\right)\right] \\
& +C_{2}\left[\left(\vec{\sigma} \cdot \vec{\sigma}^{\prime}\right) q^{2}-(\vec{\sigma} \cdot \vec{q})\left(\vec{\sigma}^{\prime} \cdot \vec{q}\right)\right],
\end{aligned}
$$

and for the $\bar{Q} q$ system is

$$
\begin{aligned}
\vec{J}_{s_{1} s_{2}} \cdot \vec{J}_{s_{3} s_{4}}= & -C_{1}\left[\left(\vec{\tau} \cdot \vec{\sigma}^{\prime}\right)\left(\vec{q} \cdot \vec{p}^{\prime}\right)-\left(\vec{\tau} \cdot \vec{p}^{\prime}\right)\left(\vec{\sigma}^{\prime} \cdot \vec{q}\right)\right] \\
& +C_{2}\left[\left(\vec{\tau} \cdot \vec{\sigma}^{\prime}\right) q^{2}-(\vec{\tau} \cdot \vec{q})\left(\vec{\sigma}^{\prime} \cdot \vec{q}\right)\right]
\end{aligned}
$$

where

$$
\begin{aligned}
C_{1} & =\frac{q^{2}}{16 m_{p} m_{l}^{3}}-\frac{\vec{p}^{\prime} \cdot \vec{q}}{8 m_{p} m_{l}^{3}} ; \\
C_{2} & =\frac{1}{4 m_{p} m_{l}}-\frac{p^{\prime 2}}{8 m_{p} m_{l}^{3}}+\frac{3 \vec{p}^{\prime} \cdot \vec{q}}{16 m_{p} m_{l}^{3}}-\frac{3 q^{2}}{32 m_{p} m_{l}^{3}} ; \\
\vec{\sigma}_{i j} & =\chi_{s_{i}}^{\dagger} \vec{\sigma} \chi_{s_{j}} \quad ; \quad \vec{\tau}_{i j}=\chi_{s_{i}}^{c \dagger} \vec{\sigma} \chi_{s_{j}}^{c},
\end{aligned}
$$

where the prime in $\vec{\sigma}$ and $\vec{\tau}$ indicates they are related to heavy currents and $m_{p}$ $\left(m_{l}\right)$ is the mass of the heavy (light) quark. 


\section{F. G. Fetter et al.}

The next step is to isolate the spin-spin interaction terms from the potential through a Fourier transform, resulting in

$$
V_{s s}=\left[a_{1}+a_{2} q^{2}+a_{3}\left(\vec{p}^{\prime} \cdot \vec{q}\right)+a_{4} p^{\prime 2}\right] \frac{\vec{\sigma}}{2} \cdot \frac{\vec{\sigma}^{\prime}}{2},
$$

where

$$
a_{1}=-\frac{8 \pi \alpha_{s}}{3 m_{p} m_{l}} \quad ; \quad a_{2}=\frac{3 \pi \alpha_{s}}{2 m_{p} m_{l}^{3}} \quad ; \quad a_{3}=-\frac{4 \pi \alpha_{s}}{m_{p} m_{l}^{3}} \quad ; \quad a_{4}=\frac{2 \pi \alpha_{s}}{m_{p} m_{l}^{3}} .
$$

\section{Perspectives}

The resulting heavy-light potential can be used to define a semi-relativistic effective meson-baryon potential which opens a perspective for future calculations like charmonium-nucleon cross sections and phase-shifts. One can also add to the potential a color confinement and a Coulombian color terms besides adopting a running coupling constant to have a more realistic model.

\section{Acknowledgments}

This work was funded by Capes and Universidade Federal do Rio Grande do Sul (UFRGS).

\section{References}

1. D. Hadjimichef, G. Krein, S Szpigel, and J. S. da Veiga, Ann. of Phys. 268 n.1, 105 (1998).

2. D. Hadjimichef, J. Haidenbauer, and G. Krein, Phys. Rev. C63, 035204 (2001).

3. D. Hadjimichef, J. Haidenbauer, and G. Krein, Phys. Rev. C66, 055214 (2002).

4. V. B. Berestetskii, E. M. Lifshitz, and L. P. Pitaevskii; Quantum Electrodynamics, Course of Theoretical Physics, Vol. 4, 2nd edn. (Elsevier, Oxford, 1982).

5. E. S. Ackleh, T. Barnes, and E. S. Swanson, Phys. Rev. D54, 6811 (1996). 